\title{
Christentum und Islam als Gegenpositionen rechtspopulistischer Identitätsstiftung. Bemerkungen zum Religionsdiskurs der AfD
}

\section{Christianity and Islam in Opposing Positions of Right-Wing Populist Identity Foundations: Remarks on the Religious Discourse in the AfD}

\section{Georg SCHUPPENER 1 (D)}

1 Prof. Dr. Dr., Univerzita sv. Cyrila a Metoda v Trnave, KGER FF, Trnava, Slowakei

\section{ORCID: G.S. 0000-0002-8945-4601}

\section{Corresponding author:} Georg SCHUPPENER,

Univerzita sv. Cyrila a Metoda v Trnave, KGER FF, Trnava, Slowakei

E-mail: georg.schuppener@ucm.sk

Submitted: 05.08.2020

Revision Requested: 06.11.2020

Last Revision Received: 07.11.2020

Accepted: 27.11.2020

Citation: Schuppener, G. (2020).

Christentum und Islam als

Gegenpositionen rechtspopulistischer Identitätsstiftung.Bemerkungen zum

Religionsdiskurs der AfD. Alman Dilive Edebiyatı Dergisi - Studien zur deutschen Sprache und Literatur, 44, 41-68.

https://doi.org/10.26650/sdsl2020-0018

\section{ABSTRACT (DEUTSCH)}

Der Beitrag befasst sich mit Wahlplakaten und Wahlprogrammen der rechtspopulistischen Alternative für Deutschland (AfD) und untersucht diese Textsorten daraufhin, welche Rolle das Thema Religion spielt. Dafür wurde ein Korpus mit Plakaten und Programmen aus dem Zeitraum der Jahre 2016-19 zugrunde gelegt. Berücksichtigt wurde dabei nicht nur die Bundestagswahl 2017, sondern auch alle Landtagswahlen in diesem Zeitraum sowie die Wahl zum Europäischen Parlament 2019. Da Wahlplakate als multimodale Texte aufgefasst werden können, wurde zunächst eine Bildrecherche durchgeführt. Diese ergab, dass nur wenige unmittelbar religionsbezogene Motive verwendet werden. Gleiches konnte für die Bildlichkeit in den Wahlprogrammen festgestellt werden. Deutlichere Ergebnisse erbrachte die Analyse der Lexik. Für beide Textsorten konnte nachgewiesen werden, dass religionsbezogene Lexik mit Islam-Bezug im Vergleich zu solcher mit Referenz zum Christentum eindeutig dominiert. Die Auswertung der Lexik belegt ferner, dass die Thematik Judentum in beiden Textsorten keine nennenswerte Rolle spielt. Hinsichtlich der Einbettung in Diskurse zeigt die Kontext- und Metaphern-Analyse, dass die Bezugnahme auf Religiöses zur Stiftung und Abgrenzung von Identität und Alterität dient. In beiden Hinsichten wird dabei lediglich auf Vordergründiges und Äußerliches referiert. Christliches als Eigenes bleibt hier gänzlich unspezifisch, Islamisches wird hingegen für ein Bedrohungsszenario genutzt. Eine Auseinandersetzung mit Glaubensinhalten findet dabei in keinem Falle statt.

Schlüsselwörter: Rechtspopulismus, Politolinguistik, Wahlwerbung, Plakate, Wahlprogramme

\section{ABSTRACT (ENGLISH)}

This paper presents a textual analysis of election posters and programs of the right-wing populist party, Alternative für Deutschland (AfD). The role of religion is examined in these texts. A corpus of posters and programs from the period 201619 were utilized for this analysis. The study covers all state elections in this period: 
the German federal elections of 2017 and the elections to the European Parliament in 2019. Since election posters can be understood as multimodal texts, image research was conducted first. This revealed that only a few religion-related motifs were used on election posters. This was also the case for the imagery in the election manifestos. The analysis of the lexicon yielded different results. For both types of texts, religion-related lexicons referring to Islam clearly dominate compared to Christianity. The lexical analysis also shows that the subject of Judaism does not play any significant role in either type of text. With regard to embedding in discourses, the context and metaphor analysis shows the references to religious issues found and delimit identity and alterity. In both cases, only the superficial and external aspects are referred to. Christianity, as an identity reference, remains completely unspecific, whereas Islam is used as a threat scenario. In not one case is there engagement with the contents of faith. The central result of the study is that right-wing populist election advertising is not about a real confrontation between Christianity and Islam. Rather, the election campaign focuses on the rejection of Islam and thus on the rejection of migration.

Keywords: Right-wing populism, political linguistics, election advertising, posters, election programs

\section{EXTENDED ABSTRACT}

In the space of just a few years, the right-wing populist party "Alternative für Deutschland" (AfD) has succeeded in gaining parliamentary seats after contesting federal and state elections. The AfD is now also represented in a large number of parliaments at the local level. Several studies on the party's policies have been published. Similarly, from a political-linguistic point of view, some aspects of the language of the AfD have been examined in great detail. Like all right-wing populist parties in Europe, the AfD has a negative view on Islam. The relationship between the chief Christian churches in Germany and the AfD is also extremely problematic. Against this background, the aim of this paper is to minutely examine how the AfD positions itself in relation to Christianity, Christian values, Christian traditions, and other aspects of Christian culture. Furthermore, the AfD's position on Christianity compared to that on Islam is considered. To the best of our knowledge, such a comparison, which highlights the differences, has so far not been done in such detail.

The paper looks at the election posters and election programs of the AfD. These two types of texts represent extremes in the way the party presents itself in its election campaigns. While election programs detail the goals of the AfD in the context of the specific election being contested, often explaining the party's objectives in an argumentative way, election posters by contrast present the programmatic goals as briefly and pointedly as possible. Both types of text are analyzed to determine the role played by religion. A corpus of posters and programs from the period 2016-19 was used for content analysis. This paper covers all state elections in this period: the German federal elections of 2017 and the elections to the European Parliament in 2019. Since election 
posters can be understood as multimodal texts, image research was conducted first. This revealed that only a few religion-related motifs were used. This was also the case for the imagery in the election manifestos. The analysis of the lexicon yielded different results. For both types of text, it could be shown that religion-related lexicons with reference to Islam clearly dominate compared to those concerning Christianity. The (few) Christian motifs found there can usually be interpreted as a means of establishing a connection to the homeland (Heimat).

The analysis of the lexis yielded clearer results. First, the word-forming production of religion-related lexis in the texts was examined. Although the general lexeme religion leads the ranking in a quantitative list, Islam-related lexemes follow almost exclusively. In the statistical analysis of the absolute frequencies of 70 religion-related lexemes, references to Islam clearly dominate for both types of texts in comparison to Christianity. Analysis of the lexemes also shows that the topic of Judaism does not play a significant role in either text type. With regard to embedding in discourses, the context and metaphor analysis shows that the reference to religious issues serves to found and delimit identity and alterity. In both respects, only the superficial and external aspects are referred to. Christianity as an identity marker remains completely unspecific in the presentation. Thus, the reference to Christian-Western values or culture, which is repeatedly emphasized in the election manifestos, remains vague in terms of content. An exact definition of this concept is missing in the AfD texts. Islamic values, on the other hand, are invoked for a threat scenario. Islam is associated with crime, terror, lack of integration, and lack of commitment to legal norms. However, there is no engagement with religious content. These findings are illustrated using selected posters as examples. Also, the paper shows that the election advertising of the AfD employs regional specifics when setting priorities in the area of religion. In this context, emotionalism and scandalmongering are used to stereotype Muslims as a significant threat. 


\section{Vorbemerkungen und Fragestellung}

Der Partei "Alternative für Deutschland“ (AfD) ist seit 2014 bei allen Wahlen auf Bundes- ${ }^{-1}$ und Landesebene der Einzug in die Parlamente gelungen. Auch auf kommunaler Ebene ist die Partei inzwischen in einem Großteil der Parlamente vertreten. Damit ist die Partei binnen kürzester Zeit (Gründung: 6.2.2013) zu einem ernstzunehmenden Faktor innerhalb der bundesdeutschen politischen Landschaft geworden.

Zur Politik der AfD gibt es inzwischen zahlreiche wissenschaftliche Analysen, (z.B. Butterwegge, Hentges \& Wiegel, 2018; Häusler, 2016) u.a. auch schon einige Untersuchungen aus politolinguistischer Perspektive (z.B. Kranert, 2018; Mathias, 2018; Niehr \& Reissen-Kosch, 2018). Politisch wird die Partei dabei als rechtspopulistisch klassifiziert. Sie gehört damit zu einer in der jüngeren Vergangenheit in Europa (aber auch darüber hinaus) verbreiteten und erfolgreichen politischen Bewegung.

Zu den verschiedenen Merkmalen rechtspopulistischer Parteien in Europa gehört insbesondere die Ablehnung von Einwanderung und - damit eng verbunden - die dezidiert negative Positionierung zum Islam, stammen doch viele der von den Rechtspopulisten abgelehnten Einwanderer aus islamischen Ländern, vor allem seit der so genannten „Flüchtlingskrise“ im Jahre 2015. Dieser Aspekt der Politik der AfD wurde in der Forschung schon mehrfach beleuchtet (z.B. Häusler, 2017, S. 70f.).

Wie das Verhältnis von AfD zum Christentum bzw. umgekehrt von Christen zur AfD ist, wird vereinzelt in der theologischen Literatur diskutiert, (z.B. Thielmann, 2017) darüber hinaus aber auch aus politologisch-soziologischer Sicht betrachtet (Püttmann, 2017; Strube, 2017).

Die großen Kirchen in Deutschland, d.h. die Evangelische Kirche in Deutschland (EKD) und die Evangelischen Landeskirchen sowie die Katholische Kirche und die verschiedenen katholischen Bistümer, haben sich mehrfach kritisch mit der AfD und ihrer Politik auseinandergesetzt und sich zum Teil klar gegen diese positioniert (Z.B. https://www.ekd. de/ekd-ratsvorsitzender-in-aller-klarheit-gegen-afd-hetze-37096.htm; https://www. vaticannews.va/de/kirche/news/2019-11/deutschland-katholikenkomitee-widerstandafd.html).

1 Bundestagswahl 2017, Wahl zum Europäischen Parlament 2014, Wahl zum Europäischen Parlament 2019. 
Auf der anderen Seite gibt es in der Partei eine Vereinigung "Christen in der AfD" (ChrAfD), die - wenngleich sie nur wenige Mitglieder und damit nur geringen Einfluss in der Partei hat ${ }^{2}$ - durchaus offensiv mit Selbstdarstellungen an die Öffentlichkeit tritt (Kuhs, 2018) und dabei motiviert, warum Christen AfD wählen (sollten). Allerdings kritisierten Politiker der AfD die Kirchen wiederholt, so beispielsweise den Einsatz der EKD für die Flüchtlingsrettung im Mittelmeer, aber auch deren Abgrenzung gegenüber rechtspopulistischen Positionen (z.B. https://www.evangelisch.de/inhalte/156631/11- 062019/afd-politiker-um-hoecke-werfen-ekd-politisierung-vor; https://www.nwzonline.de/ meinung/berlin-analyse-afd-im-clinch-mit-den-kirchen_a_50,3,1227031897.html). Das Verhältnis zwischen den großen christlichen Kirchen in Deutschland und der AfD ist also insgesamt äußerst problematisch.

Vor diesem Hintergrund erscheint es sinnvoll, genauer zu untersuchen, wie sich die AfD zum Christentum, zu christlichen Werten, christlichen Traditionen und anderen Aspekten christlicher Kultur verhält und positioniert. Ferner ergibt sich die Frage, wie die AfD ihre Position zum Christentum derjenigen zum Islam gegenüberstellt. Ein Vergleich kann hier die Unterschiede pointiert herausarbeiten. Bisher wurden diese Gesichtspunkte noch nicht eingehender untersucht, ${ }^{3}$ insbesondere nicht aus politolinguistischer Perspektive, d.h. speziell im Hinblick auf die Diskurse in der Außenkommunikation der AfD.

Da gerade im Rahmen des Wahlkampfes Positionen von Parteien besonders deutlich artikuliert und hinterfragt werden, sollen im Folgenden Texte aus dem Umfeld von Wahlkämpfen ausgewertet werden, und zwar dahingehend, welche Rolle und welche Funktion die AfD dem Christentum und dem Islam im weitesten Sinne zuweist und ob und wie dies in diesen Texten explizit wird. Da rechtspopulistischen Bewegungen bisweilen auch (offene oder latente) antisemitische Tendenzen vorgeworfen werden, (Grigat, 2017) soll ferner auch ein Blick darauf gerichtet werden, ob und wie das Judentum und jüdisches Leben bei der AfD thematisiert werden.

\section{Korpus}

Um bei einer solchen Untersuchung aussagekräftige und nach Möglichkeit repräsentative Befunde zu erzielen, ist es notwendig, ein geeignetes Korpus zugrunde zu

2 Die Anzahl der Mitglieder wird auf 125 Personen geschätzt (Stand: Ende 2017). (https://de.wikipedia.org/ wiki/Christen_in_der_AfD)

3 Auf die Gegenüberstellung in der ebenfalls rechtspopulistischen FPÖ geht Demčišák (2019, S. 164f.) kurz ein. 
legen und dieses zuvor nach klaren Kriterien zu erstellen. Ein solches Korpus soll allein Primärtexte enthalten, also solche Texte, die auf die AfD selbst zurückgehen. Darüber hinaus beschränkt sich das Korpus auf schriftliche Texte, die für eine breitere Öffentlichkeit bestimmt sind und damit das Außenbild der Partei präsentieren.

Ausgewählt wurden hierfür die folgenden beiden Textsorten: Wahlplakate und Wahlprogramme. Diese beiden Textsorten repräsentieren Extreme in der Form der wahlwerblichen Selbstdarstellung einer jeden Partei:

Während Wahlprogramme die Ziele einer Partei - bezogen auf die kommende Wahlperiode - mehr oder minder ausführlich darstellen und häufig auch argumentativ erläutern, kommt es bei Wahlplakaten darauf an, die programmatischen Ziele möglichst kurz und zugespitzt zu präsentieren. Denn Wahlprogramme dienen der detaillierten Selbstdarstellung, die oftmals so ausführlich ist, dass es neben einer Langfassung zum besseren und übersichtlicheren Verständnis für breitere Wählerschichten noch eine Kurzfassung mit den wichtigsten Forderungen und Eckpunkten gibt. Wahlplakate hingegen werden in der Regel nur en passant wahrgenommen, bisweilen nur für Sekunden oder Sekundenbruchteile, so dass sich die sprachliche und bildliche Botschaft auf das Wesentliche, d.h. gemeinhin eine Kernaussage, begrenzen muss ${ }^{4}$.

Bei der Betrachtung dieser beiden wahlkampftypischen und zugleich doch recht unterschiedlichen Textsorten ist zu erwarten, dass sich - sofern vorhanden Schwerpunkte im Religionsdiskurs identifizieren lassen.

Für das Korpus, das sich auf Grund der Auswertung zweier Textsorten eigentlich aus zwei Teilkorpora zusammensetzt, wurde folgendes Material ausgewählt:

Das Korpus von Wahlplakaten der AfD umfasst Plakate aus den Jahren 2016 bis 2019. Hierbei wurden sowohl die Plakate zur Bundestagswahl 2017, der wichtigsten Wahl im politischen Leben der Bundesrepublik Deutschland, als auch zu mehreren Landtagswahlen in deren zeitlichem Umfeld (2016: Baden-Württemberg, Berlin, Mecklenburg-Vorpommern, Rheinland-Pfalz, Sachsen-Anhalt, 2017: Niedersachsen, Nordrhein-Westfalen, Saarland, Schleswig-Holstein, 2018: Bayern, Hessen, 2019:

4 Die Forschungslage zu Texten von Wahlplakaten ist bislang recht übersichtlich. Es existieren kaum mehr als ein Dutzend einschlägiger Studien (u.a. Khodyeyev, 2016; Michel \& Pappert, 2018a, 2018b; Müller, 1978, 2001; Pappert, 2016; 2017; Poier, 2004; Schuppener, 2018; 2019). 
Brandenburg, Sachsen, Thüringen ${ }^{5}$ ) aufgenommen, ferner die Plakate zur Wahl zum Europäischen Parlament im Jahre 2019. Nicht im Korpus berücksichtigt wurden Plakate zu Wahlen auf kommunaler Ebene, und zwar deswegen, weil sie einerseits in der Regel stärker Personen denn Programmatik in den Vordergrund stellen und sie andererseits nachträglich nur schwer zu erheben sind.

Die Plakate des Korpus wurden im Zeitraum von Dezember 2018 bis Mai 2020 mit Bildrecherchen im Internet erschlossen, vornehmlich von offiziellen Seiten der AfD ${ }^{6}$. Auf diese Weise konnte ein hoher Grad an Authentizität ${ }^{7}$ und Repräsentativität erzielt werden, auch wenn eine vollständige Erfassung aller Plakate aus den betrachteten Jahren nicht gewährleistet werden kann.

Insgesamt umfasst das Korpus 160 thematische Wahlplakate, ${ }_{1}^{8}$ davon $124 \mathrm{zu}$ den berücksichtigten Landtagswahlen, 26 zur Bundestagswahl und 10 zur Wahl zum Europäischen Parlament.

Für die Analyse muss berücksichtigt werden, dass Wahlplakate multimodale Texte darstellen, nämlich Kombinationen aus Sprache und Bild, wobei auch die Farbwahl und die Typografie zu beachten sind. Für die Analyse der Lexik spielt die Bildlichkeit zwar nur eine sekundäre, illustrative Rolle. Für die Diskurse hingegen kann die Bildkomponente eine wichtige Funktion besitzen.

Das Korpus der Wahlprogramme umfasst alle Wahlprogramme zu den oben genannten Wahlen sowie zusätzlich das zur Wahl zur Bremischen Bürgerschaft 2019. Der Umfang der insgesamt 17 Wahlprogramme ist dabei durchaus unterschiedlich: So umfasste das ausführliche Wahlprogramm zur Landtagswahl in MecklenburgVorpommern 2016 lediglich 22 Seiten, dasjenige zur Landtagswahl in Bayern 2018 hingegen ganze 100 Seiten. Auch wenn durch unterschiedliches Druckformat und unterschiedlichen Satz die Vergleichbarkeit nur bedingt gegeben ist, zeigt sich doch eine große Spannweite der Ausführlichkeit in der Darstellung politischer Ziele. Die Gesamtzahl

5 Die Plakate zur Wahl zur Bremischen Bürgerschaft 2019 wurden nicht in das Korpus aufgenommen, da die Partei hier im Wesentlichen auf thematische Plakate zurückgriff, die bereits bei der Bundestagswahl 2017 Verwendung fanden.

6 Bei den in diesem Artikel verwandten Abbildungen wurden aus technischen Gründen z.T. andere Quellen genutzt.

7 Es sei hier darauf hingewiesen, dass im Internet zahlreiche nicht von der AfD autorisierte Plakate kursieren, teils von Unterstützern der Partei, teils z.B. als Karikaturen von Gegnern der AfD.

Plakate der Wahlkreiskandidaten wurden nicht berücksichtigt. 
der Seiten aller hier betrachteten Wahlprogramme lag bei 1060, der Durchschnitt damit bei 62,35 Seiten.

Im Unterschied zu allen anderen hier betrachteten Programmen, die jeweils den Begriff Wahlprogramm im Titel führen, ist dasjenige zur Landtagswahl in Sachsen 2019 als Regierungsprogramm betitelt, was durchaus auch den Anspruch der Partei in diesem Bundesland verdeutlicht.

Anders als auf den Wahlplakaten spielen Abbildungen in den Wahlprogrammen nur eine untergeordnete Rolle. Neben den Titelbildern finden sich bisweilen Piktogramme, die auf Themenbereiche verweisen, und in einzelnen Programmen auch Bilder, die die Kapitel einleiten. Die sprachlichen Inhalte bilden hier jedoch den Schwerpunkt, während Bilder und andere grafische Elemente in ihnen zwar in unterschiedlicher Zahl vorkommen, meist jedoch lediglich akzidentielle, d.h. ausschmückende, illustrierende oder strukturierende Funktion haben. Reziprok monosemierende Sprache-BildKombinationen, d.h. solche Verknüpfungen von Sprache und Bild, bei denen nur durch die Kombination beider Komponenten eine Botschaft/Aussage verständlich wird, finden sich in den Wahlprogrammen im Unterschied zu den Wahlplakaten nicht. Insofern unterscheiden sich die Textsorten auch im Hinblick auf ihren Grad an Multimodalität.

\section{Analyse}

Um Bezüge zu einem Religionsdiskurs identifizieren zu können, wurde folgendermaßen auf zwei Ebenen verfahren:

Auf der Bildebene wurde nach christlichen, jüdischen und muslimischen religiösen Motiven gesucht (z.B. Abbildungen von Gotteshäusern, religiöse Symbole, Personen, die durch eine bestimmte Bekleidung eindeutig einer Religion zuzuordnen sind etc.). Auf Grund der oben bereits angedeuteten Spezifik der beiden Textsorten bzw. Teilkorpora waren bei den Wahlplakaten weitaus mehr Bilder zu prüfen als bei den Wahlprogrammen.

Auf der sprachlichen Ebene wurde eine elementar statistische Auswertung der Lexik vorgenommen (d.h. die Feststellung der absoluten/relativen Häufigkeiten). Gesucht wurde dabei u.a. nach den folgenden Lexemen: 
- Christ, Christentum, christlich, katholisch, evangelisch, Kirche, kirchlich,

- Jude, Judentum, jüdisch, Synagoge,

- Muslim/Moslem, Islam, muslimisch, islamisch, islamistisch, Moschee,

- Religion, religiös, Glaube, glauben.

Berücksichtigt wurden dabei auch zugehörige Wortbildungen, insbesondere Komposita. Darüber hinaus erfolgte eine Durchsicht der Texte auf Begriffe, die eindeutig oder mutmaßlich einen religiösen Bezug besitzen, wie beispielsweise Kreuz, Kippa, Burka, schächten usw. Auf diese Weise wurde insgesamt nach 70 religionsbezogenen Lexemen im Korpus gesucht.

Betrachtet man nun zunächst die Bildebene auf den Wahlplakaten, so ist das Ergebnis recht überschaubar:

Zwei Plakate aus dem Bundestagswahlkampf 2017 thematisieren Muslimisches: Auf einem der Plakate ist ein bis auf einen Sehschlitz verschleierter Frauenkopf, also die Trägerin eines Niqabs, abgebildet. Auf einem zweiten Plakat sieht man ebenfalls eine Niqab-Trägerin und im Hintergrund einen älteren Mann mit Vollbart und islamischer Gebetsmütze (sog. Takke) ${ }^{9}$.
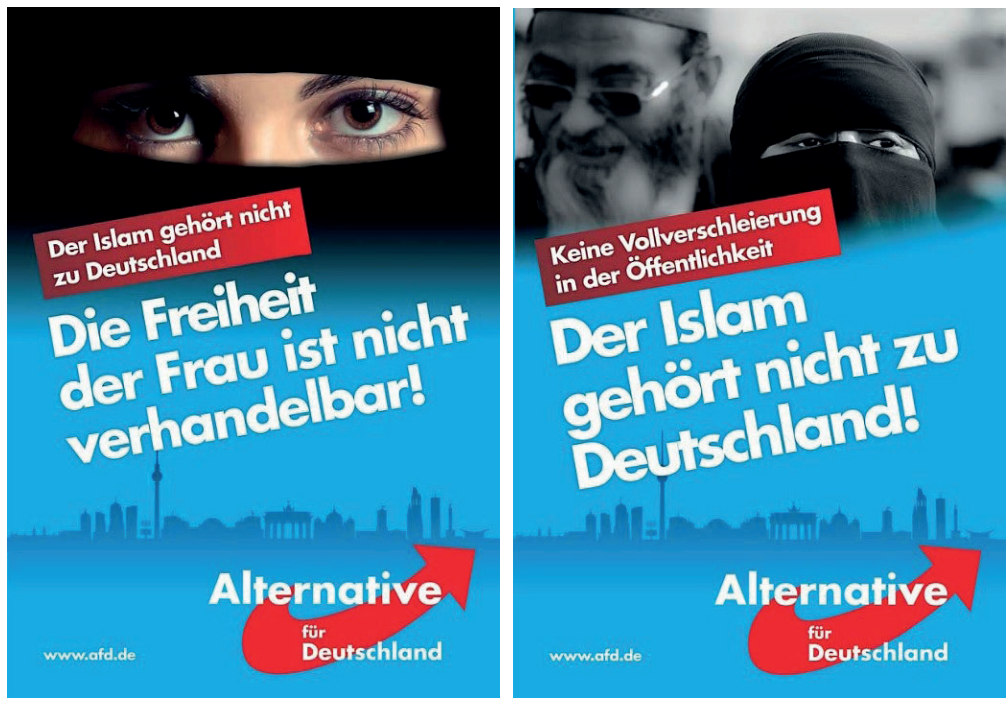

Abb. 1 + 2: Plakate zur Bundestagswahl 2017

9 Die Differenzierung zwischen Burka und Niqab ist landläufig wenig bekannt, so dass meist nur von Burka gesprochen wird. 
Aus den Landtagswahlkämpfen in Bayern, Hessen und Nordrhein-Westfalen kann jeweils ein Plakat mit (tatsächlichem oder potenziellem) christlichem Bezug identifiziert werden: Das bayerische Plakat zeigt ein Flurkreuz in Form eines überdachten Kruzifixes vor Alpenhintergrund, das Plakat aus Hessen einen hölzernen Kreuzanhänger, der auf einem aufgeschlagenen Buch, mutmaßlich einer Bibel liegt; das NRW-Plakat bildet schließlich im Hintergrund den Kölner Dom ab ${ }^{10}$.
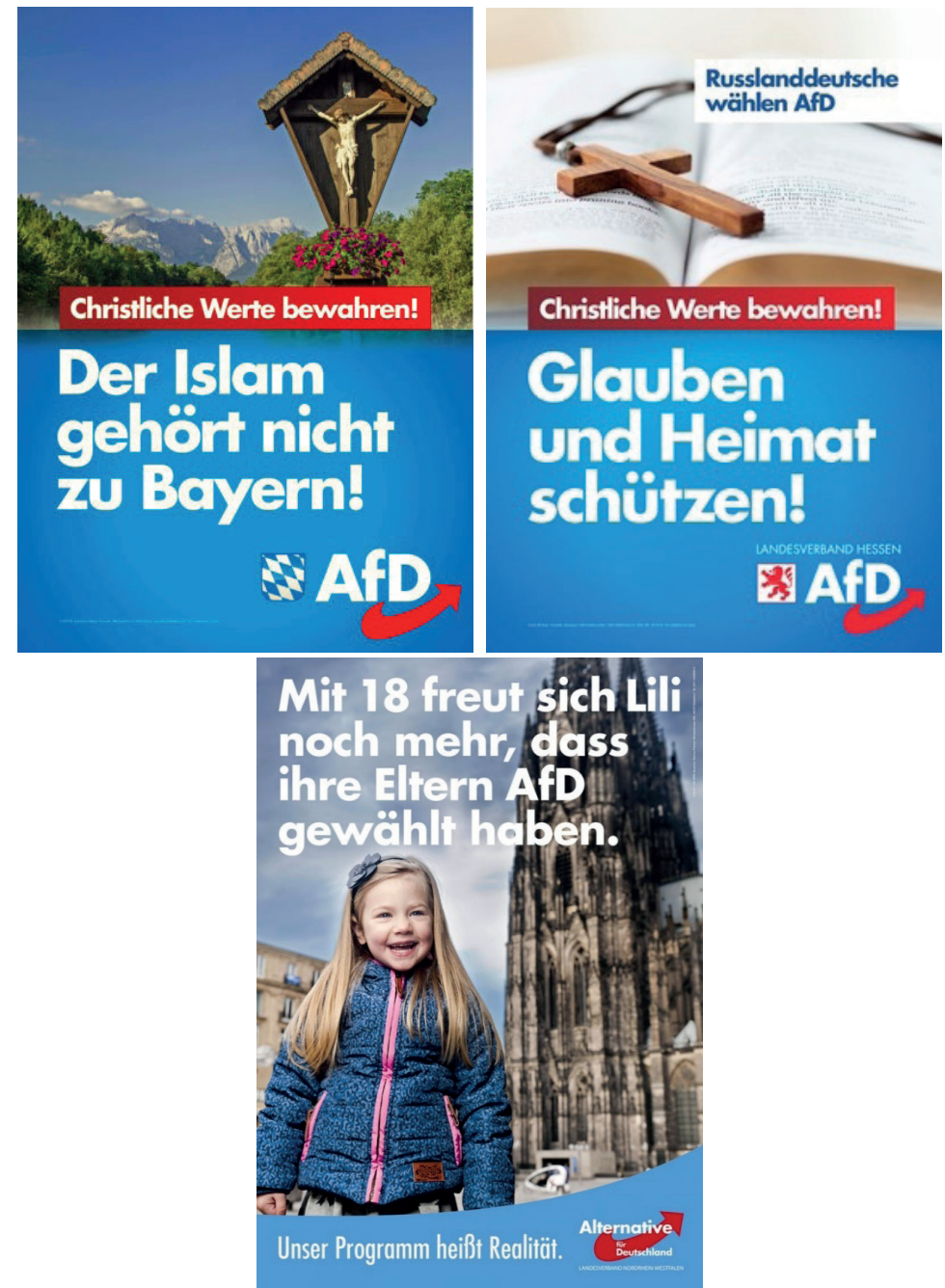

Abb. 3 + 4 + 5: Plakate zu den Landtagswahlen Bayern 2018, Hessen 2018 und NordrheinWestfalen 2017

10 Dass der Dom hier von der Intention her wohl weniger als religiöses Bauwerk, sondern als Marker für den Ort der sexuellen Übergriffe von Migranten in der Silvesternacht 2015/16 (Umfeld des Kölner Hauptbahnhofes, sog. Domplatte) genutzt wird, soll zunächst unberücksichtigt bleiben. 
Bilder mit Bezug zum Judentum finden sich überhaupt nicht. Bezogen auf die Gesamtmenge der Plakate machen damit solche mit einem religiösen Motiv kaum mehr als $3 \%$ aus.

Bei den Wahlprogrammen finden sich die folgenden Abbildungen, die hier relevant sind: Im Programm zur Landtagswahl 2018 im Freistaat Bayern ist dem Titelblatt des Kapitels 2 „Recht und Inneres“ ein Bild der Türme der Münchener Frauenkirche sowie der Mariensäule hinterlegt (AfD Bayern, 2018, S. 16). Das Wahlprogramm zur Landtagswahl 2018 in Hessen zeigt auf dem Titelblatt im Vordergrund das Gebäude des Hessischen Landtages, im Hintergrund ist die benachbarte neugotische Wiesbadener Marktkirche zu erkennen (AfD Hessen, 2018, S. 1). Ferner ist auf der Titelseite des Kapitels 15 "Kunst, Kultur und Medien" der Limburger Dom und auf der Lahnbrücke im Vordergrund die Statue des Brückenheiligen Johannes Nepomuk zu sehen (AfD Hessen, 2018, S. 85). Auf dem Titelblatt des Wahlprogrammes der AfD zur Landtagswahl in Nordrhein-Westfalen 2017 findet sich u.a. ein Bild von Köln, das durch den Dom geprägt ist, ferner wird der inhaltliche Teil des Programmes mit einem Bild des Aachener Doms einschließlich der benachbarten Kirche St. Foillan beschlossen (AfD Nordrhein-Westfalen, 2017, S. 1, 81). Im Wahlprogramm zur Landtagswahl in Rheinland-Pfalz 2016 schließlich enthält das Kapitel 10 „Infrastruktur und ländlicher Raum" ein kleines Bild einer Kirche aus dem ländlichen Raum (AfD Rheinland-Pfalz, 2016, S. 24f.).

Angesichts der Tatsache, dass die Wahlprogramme schriftlastig sind und insgesamt nur wenige Abbildungen aufweisen, mag der Befund, dass immerhin vier von 17 Programmen derartige Bilder aufweisen, auf den ersten Blick überraschend sein. Berücksichtigt man überdies die Positionierung der Bilder, so kann diese zum überwiegenden Teil als exponiert/prominent bezeichnet werden. Aus der Auswahl der abgebildeten Objekte ergibt sich aber auch bereits ein Schluss auf die Funktion dieser Bilder. Mit Ausnahme der kleinen Abbildung im rheinland-pfälzischen Wahlprogramm zeigen alle anderen identifizierten Abbildungen prominente Bauwerke. Da sie allgemein bekannt sind, brauchen sie auch nicht durch einen Untertitel benannt zu werden. Überdies stammen alle abgebildeten Bauwerke aus den jeweiligen Bundesländern. Es liegt nahe, dass die Abbildungen einen Heimatbezug herstellen sollen. Damit dienen sie primär der Konstruktion einer (Landes-)Identität. Dass dabei Heimat und Identität als christlich geprägt (im Sinne von christlichem Abendland) verstanden werden, mag auch daraus erschlossen werden, dass Abbildungen mit jüdischem oder islamischem Bezug gänzlich fehlen. Hierbei ist allerdings zu berücksichtigen, dass beispielsweise Synagogen 
oder Moscheen weit weniger im allgemeinen Bewusstsein präsent und derartige Bauwerke daher kaum bekannt sind. Inwieweit in den betreffenden Programmen also ein dezidiert christlicher Bezug intendiert ist, lässt sich somit nicht abschließend beurteilen.

Einen genaueren Einblick auf die Zugriffe der AfD auf Religionsdiskurse verspricht hingegen die lexikalische Analyse. Die Ergebnisse hinsichtlich der Sprache auf Wahlplakaten können wie folgt dargestellt werden (in alphabetischer Reihenfolge):

Burka 4x (davon 1 x bei Landtagswahlen), christlich 2x (2), Glaube 1x (1), Halal 2x (2), Islam 6x (2), islamfrei 1x (1), kopftuchfrei 1x (1), Kreuzpflicht 1x (1), muslimisch 1x (1), schächten $2 x(2)$.

Die Wahlprogramme wurden ebenfalls komplett auf religiöse Lexik durchsucht. Das Ergebnis zeigt, dass die Thematik der Religion durchaus eine relevante Rolle innerhalb der Programme spielt, zumindest wenn man die Produktivität von einschlägigen Lexemen hinsichtlich der Wortbildung als Indiz zugrunde legt:

So weist Religion von der erfassten Lexik die größte Zahl an Wortbildungen auf, nämlich zwölf: Religionsausübung, Religionsbehörde, Religionsfreiheit, Religionsgemeinschaft, Religionsgeschichte, Religionsgesellschaft, Religionsgruppe, Religionskritik, Religionskunde, Religionsrabatt, Religionsunterricht, religiös. Es folgen (in zahlenmäßig absteigender Reihenfolge) Kirche mit acht (kirchenähnlich, Kirchenasyl, Kirchengemeinde, Kirchengemeinschaft, Kirchenmitglied, Kirchenrepräsentant, Kirchensteuerzahler, kirchlich), Islam mit sieben (islamisch, Islamisierung, Islamismus, Islamist, islamistisch, Islamunterricht, Islamverband), Moschee mit sechs (Moscheebau, Moscheebesuch, Moscheebesucher, Moscheegemeinde, Moscheeverband, Moscheeverein) und Glaube mit vier Wortbildungen (Glaubensfreiheit, Glaubensgemeinschaft, Glaubenspraxis, Glaubensrichtung). Alle anderen Lexeme mit Religionsbezug weisen keine oder lediglich ein oder zwei zugehörige Wortbildungen auf.

Innerhalb des religionsbezogenen Wortschatzes lassen sich aus der Anzahl der Belege leicht Schwerpunkte identifizieren. Zwar führt die Liste der absoluten Häufigkeiten das Adjektiv religiös mit 81 Belegen an, es folgen dann aber (in absteigender Reihenfolge):

islamisch (59), Islam (56), Religion (41), muslimisch (32), Muslim(a) (32), christlich (28), Imam (25), Moschee (24). 
Die starke Fokussierung auf Islam-bezogene Lexik ist dabei offenkundig.

Während Religion, Glaube und die zugehörigen Wortbildungen für sich betrachtet neutral sind, lässt sich ein großer Teil der religiösen Lexik eindeutig einer bestimmten Religion zuordnen ${ }^{11}$. Das dabei entstehende Bild verdeutlicht klar, wo die AfD programmatische Schwerpunkte hinsichtlich religiösen Themen setzt: Es stehen sich nämlich 270 lexikalische Belege zum Islam, 76 zum Christentum und 5 zum Judentum gegenüber.

Judentum und Jüdisches werden also von der AfD in den Wahlprogrammen fast nie in den Blick genommen. Die Lexeme Jude (2 Belege), Judentum (2), jüdisch (1) liegen in der Vorkommenshäufigkeit im Korpus deutlich hinter solchen (ansonsten peripheren) Begriffen wie Niqab (5) oder Kopftuchverbot (8). Bemerkenswerterweise wird im Zusammenhang mit dem Judentum suggeriert, dass Antisemitismus ein allein mit dem Islam verbundenes Problem sei. So wird im Europawahlprogramm 2019 die Forderung „Antisemitismus in Wort und Tat muss konsequent bekämpft werden." im Kapitel „Islam Gefahr für Europa" behandelt (Alternative für Deutschland, 2019, S. 52). Derselbe Konnex wird im Programm zur Wahl zum Abgeordnetenhaus Berlin 2016 hergestellt, dort sogar noch expliziter: „Insbesondere ein wachsender Antisemitismus unter Muslimen bereitet Anlass zur Sorge" (AfD Berlin, 2016, S. 21). Ansonsten spielt das Thema Antisemitismus in den Programmen keine Rolle.

Einander gegenübergestellt werden also gemeinhin Christentum und Islam, wobei wie das Verhältnis der Belege deutlich zeigt - die Aufmerksamkeit eindeutig mehr dem Islam gilt als dem Christentum.

Welcher Art die jeweilige Befassung ist, lässt sich aber aus der bloßen lexikalischen Analyse nicht valide erschließen. Zwar geben die Belege für Begriffe wie Burkaverbot, Kopftuchverbot oder Vollverschleierungsverbot bereits den Hinweis auf einen speziellen Diskurs in der Auseinandersetzung mit Islam und islamischer Kultur - und ähnliches gilt auch für die lexikalische Gruppe Islamisierung, Islamist, islamistisch, doch der Großteil der Begrifflichkeit gibt durch seine reine Belegung keine direkten Hinweise auf der Art und Weise, wie sie seitens der AfD ausgedeutet wird und welche Diskurse hierbei angesprochen werden.

11 Während Burka (3 Belege) und Niqab (5 Belege) eindeutigen Islambezug besitzen, ist dies zumindest formal bei Kopftuch (17 Belege) nicht so. Durch den Kontext ist aber in allen Fällen klar, dass es hierbei um das Kopftuch als islamisches religiöses Symbol geht. 
Noch weniger Aufschlüsse über die konkrete Einbindung in Diskurse bietet die erhobene Lexik mit Bezug zum Christentum, nämlich Lexeme wie Christ, Kreuz oder die Wortbildungen zu Kirche.

Der Religionsdiskurs wird also bei der AfD zugespitzt mit den Polen Christentum und Islam geführt. Betrachtet man zunächst die Verwendungskontexte islambezogener Lexik, so stellt man rasch fest, dass der Islam und muslimisches Leben als fremd und als Bedrohung dargestellt werden, unvereinbar mit Traditionen und überkommenen Werten in Deutschland. Auf Wahlplakaten wird dies pointiert mit den assertiven Slogans "Der Islam gehört nicht zu Deutschland" (u.a. Abb. 1 + 2) betont - einer Negation der Feststellung, die erstmals im Jahre 2006 der damalige Bundesminister des Innern, Wolfgang Schäuble, und dann - deutlich öffentlichkeitswirksamer - im Jahre 2010 der damalige Bundespräsident Christian Wulff äußerte (https://www.deutschlandfunkkultur. de/die-geschichte-eines-satzes-der-islam-gehoert-zu-deutschland.1895.de.html?dram: article_id=308696). Enggeführt wird diese Behauptung mit den Themen Vollverschleierung/Burka/Frauenrechte. Allein drei der 26 thematischen Plakate zur Bundestagswahl 2017 führen explizit diesen Satz, weitere Plakate modifizieren ihn („,'Der Islam?' Passt nicht zu unserer Küche.") oder transportieren latent diese Botschaft, wie beispielsweise in Form einer Ablehnung einer EU-Mitgliedschaft der Türkei (Europa endet am Bosporus! Die Türkei gehört nicht in die EU).
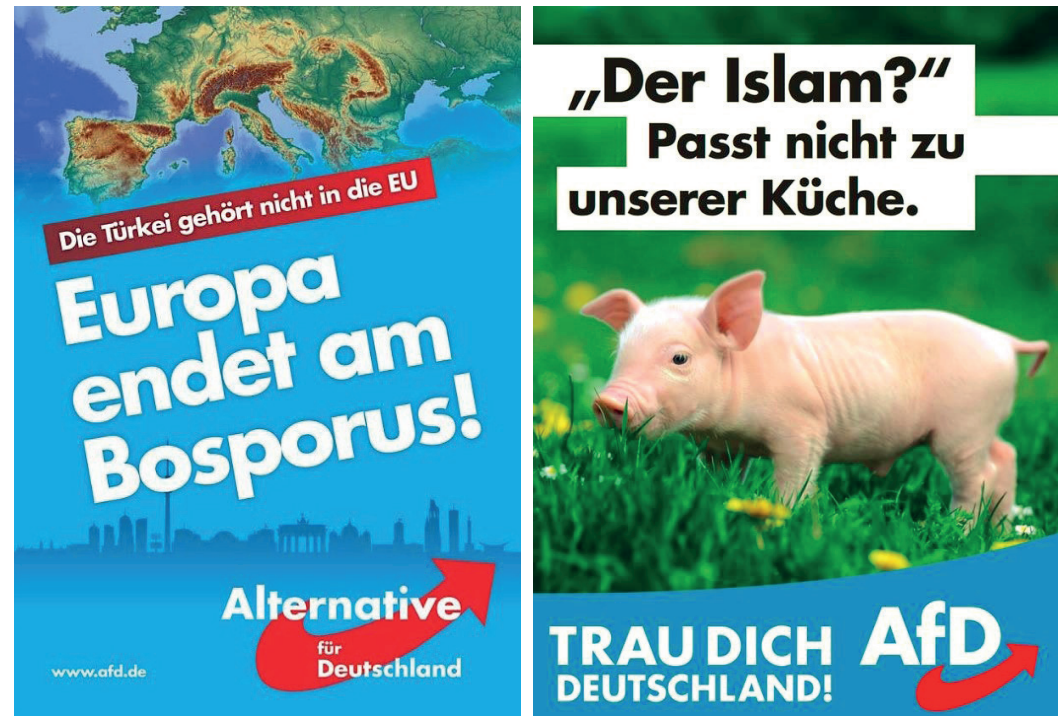

Abb. 6 + 7: Plakate zur Bundestagswahl 2017 
Diese mehr oder weniger abstrakten Feststellungen werden auf anderen Plakaten personalisiert und damit konkretisiert, wie die folgenden Beispiele belegen:

Vor dem Hintergrund einer stark von Menschen mit Migrationshintergrund und damit multikulturell geprägten Gesellschaft in Berlin warb die AfD bei der Wahl zum Abgeordnetenhaus 2016 mit einer Sprache-Bild-Kombination, die suggeriert, dass ein Betroffener vor dem Islam warnt (vgl. Abb. 8).

In der als Zitat gekennzeichneten Aussage wird der typografisch durch Kursivschreibung hervorgehobene Zentralbegriff Islam latent als Gefahr dargestellt. Dabei bleibt die Gefahrenwarnung „ich weiß, was der Islam bedeuten kann“ allerdings bewusst vage. Durch die Formulierung in der 1. Person Singular wird ein Bezug zu dem abgebildeten jungen Mann hergestellt. Dass dieser offenkundig einen Migrationshintergrund hat, legt nicht nur das Bild nahe, sondern auch der Appell an die "deutschen Freunde" im Zitat. Diese Zuschreibung soll der Aussage über den Islam Authentizität und Glaubwürdigkeit verleihen. Auch wenn mit den „deutschen Freunde[n]“ über das Personalpronomen wir (bzw. uns) Gemeinschaft hergestellt und Berlin als „unsere Heimat" bezeichnet wird, erweist sich doch der intensionale Kern der Aussage als gegen Migration gerichtet. Denn es wird ein begrifflicher und inhaltlicher Kontrast von Islam als Gefahr und bedrohter Heimat (= Berlin) hergestellt. Der Schutz richtet sich damit gegen den Islam, der in Opposition zu Heimat steht, damit fremd ist und diese bedroht.

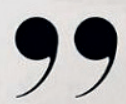

Ich wähle die AfD, weil ich weiß, was der Islam bedeuten kann, wenn wir nicht aufpassen. Meine deutschen Freunde, laßft uns diese tolle Stadt Berlin, unsere Heimat, beschützen!

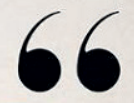

AFD - UNBEQUEM. ECHT. MUIIG.

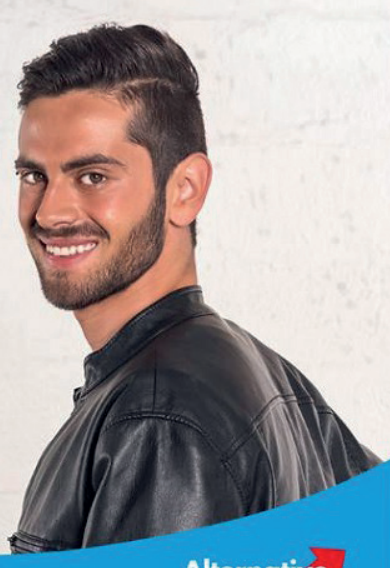

Alternative

I. Cundeutschiland

Abb. 8: Plakat zur Landtagswahl Berlin 2016 
Ohne dass der Islam überhaupt explizit erwähnt wird, vermittelt auch das folgende Plakat mit der Aufschrift „Für meine Rechte - natürlich AfD“ und der Abbildung eines den Betrachter ansehenden Lammes zur Landtagswahl im Freistaat Sachsen 2019 latent eine antiislamische Botschaft. Der informierte Betrachter erkennt, dass sich das Plakat gegen (islamisches) Schächten richtet. Das Schaf verkörpert hier das Subjekt, das in seinen (Tier-)Rechten durch den Islam bedroht wird. Die Abbildung nutzt das so genannte Kindchen-Schema und emotionalisiert damit die Ablehnung der unausgesprochenen islamischen Praxis.

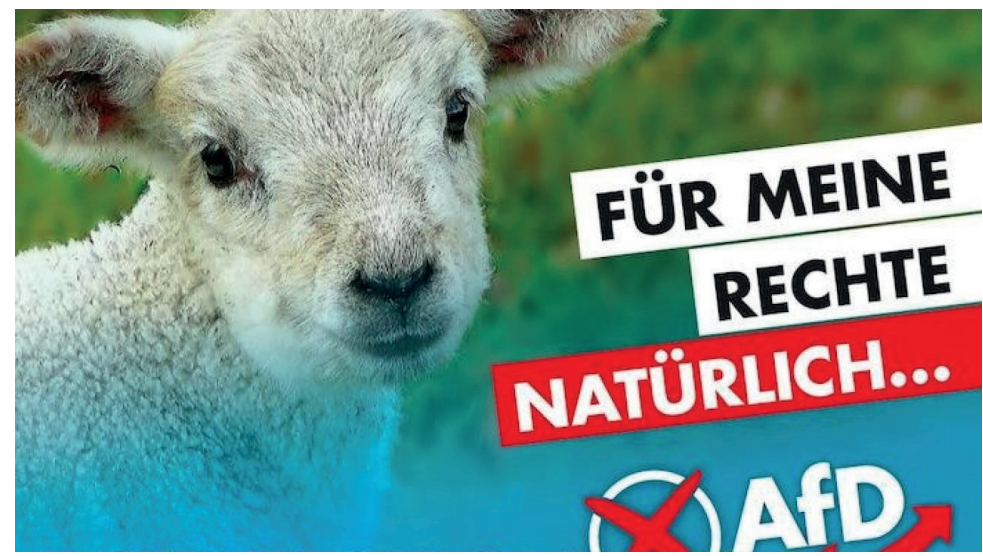

Abb. 9: Plakat zur Landtagswahl 2019 im Freistaat Sachen

Elaborierter ist naheliegenderweise die Positionierung der AfD in den Wahlprogrammen. Hier werden der Islam und muslimisches Leben durchweg als fremd und häufig auch als gefährlich und bedrohlich dargestellt. Dies manifestiert sich insbesondere in Symbolen muslimischen Lebens, die als intendierte Unterdrückung von Traditionen und Freiheitsrechten ausgedeutet werden. So wird im Programm zur Bayerischen Landtagswahl behauptet, dass Minarette „ohne jede Ausnahme ein Symbol des islamischen Herrschaftsanspruchs darstellen" und dass eine "Islamisierung des öffentlichen Raums" durch Minarette, Muezzinrufe und Vollverschleierung drohe (AfD Bayern, 2018, S. 47). Analoge Bespiele lassen sich aus nahezu allen 17 betrachteten Programmen anführen ${ }^{12}$.

In einigen Programmen wird die klar antiislamische Positionierung der AfD dadurch deutlich, dass sogar Kapitel oder Unterkapitel der Problematik „Islam“ gewidmet werden.

12 Ausnahme ist das sehr kurze Programm zur Landtagswahl in Rheinland-Pfalz 2016, in dem der Religionsdiskurs eine nur marginale Rolle spielt (AfD Rheinland-Pfalz, 2016). 
So enthält beispielsweise das Programm zur Bundestagwahl 2017 ein eigenes Kapitel "Der Islam im Konflikt mit der freiheitlich demokratischen Grundordnung", (Alternative für Deutschland, 2017, S. 33ff.) ferner mehrere Unterkapitel, die sich mit diversen Problemen im Zusammenhang mit muslimischem Leben und muslimischer Religionsausübung befassen (Alternative für Deutschland, 2017, S. 45). Im Programm zur hessischen Landtagswahl 2018 tituliert das Unterkapitel 15.2 mit "Gefahren durch die Ausbreitung des Islam“ (AfD Hessen, 2018, S. 86). Noch allgemeiner wird als eigenes Kapitel im Europawahlprogramm „Der Islam als Gefahr für Europa“ dargestellt. Hier wird u.a. das islamische Rechtsverständnis als „totalitär“ bezeichnet, von einem „imperialistische[n] Islam" gesprochen und in diesem Zusammenhang u.a. eine „ständig drohende Terrorgefahr" ausgemacht (Alternative für Deutschland, 2019, S. 51f.).

Für politische Diskurse ist häufig die Nutzung von spezifischer Metaphorik typisch. Dies ist auch hier der Fall. Insbesondere die Kriegs- bzw. Verteidigungsmetaphorik spielt in der Befassung mit dem Thema „Islam“ eine signifikante Rolle. So wird im Programm zur Europawahl 2019 als Ziel formuliert, „Europa gegen einen Islam [zu] verteidigen“, der als „imperialistisch[...]" und „aggressiv vordringend[...]” charakterisiert wird (Alternative für Deutschland, 2019, S. 37, 51). Im Programm zur sächsischen Landtagswahl 2019 heißt es explizit: „Der politische Islam ist eine totalitäre Ideologie, die unserer Kultur feindlich gegenüber steht" (AfD Sachsen, 2019, S. 34). Ebenso passt hierzu die wiederholte Behauptung, die demokratische Grundordnung werde durch Muslime „bekämpft" (AfD Hessen, 2018, S. 86). Daraus folge umgekehrt, „Islamismus bekämpfen“ zu müssen (AfD Mecklenburg-Vorpommern, 2016, S. 13).

Eng verbunden ist damit ein Bedrohungsszenarium, zu dessen Gefahren u.a. die „Unterdrückung der Frauen“ zähle (AfD Mecklenburg-Vorpommern, 2016, S. 13). Dezidierter werden die negativen Auswirkungen im Programm für die niedersächsische Landtagswahl 2017 beschrieben:

Zunehmende öffentliche Unsicherheit, Import ethnischer und religiöser Konflikte, ein erhöhtes Risiko terroristischer Anschläge, eine erhöhte Kriminalitätsrate sowie die Missachtung der Staatsorgane und die Geringschätzung der Werte und Normen des Gastlandes sind Folgen der Zuwanderung aus fremden, insbesondere muslimischen Kulturen. (AfD Niedersachsen, 2017, S. 49) ${ }^{13}$

13 Auf derselben Seite werden noch zahlreiche weitere negative Aspekte des Islams dargestellt. 
Im Programm zur Landtagswahl im Saarland 2017 findet sich folgende Feststellung: „Die Bedrohungslage durch islamistische Terrorangriffe ist dramatisch" (AfD Saarland, 2017, S. 22).

Weit weniger präsent im Diskurs ist hingegen Christliches. Zudem ist hier die Position der AfD ambivalent: Einerseits beruft sich die Partei gerne auf "christliche Werte" als Basis abendländischer Kultur und als Teil der deutschen oder regionalen Identität, und zwar in Opposition zum Fremden, zum Islam, wie beispielsweise auf dem bayerischen Plakat (Abb. 3) in der Sprache-Bild-Kombination gut erkennbar ist. Christliche Werte werten mit (stereotyp alpenländischer) Heimat gleichgesetzt, wobei der Bezug zum Christentum durch das Kruzifix auch bildlich expliziert wird. Aus dieser Sprache-Bild-Kombination wird die Conclusio „Der Islam gehört nicht zu Bayern“ abgeleitet.

Vergleichbar ist die Botschaft auf dem hessischen Plakat (Abb. 4), das sich mutmaßlich eher an eine protestantische (russlanddeutsche) Wählergruppe richtet. Aufgeschlagene Bibel und Anhängekreuz hinterlegen den Begriff christliche Werte. Wodurch diese bedroht werden, wird nicht erläutert, vielmehr muss der Betrachter aus dem Slogan "Glauben und Heimat schützen!" eine Bedrohungssituation erschließen und speziell auf Nicht-Christliches als Bedrohung schließen.

Was christliche Werte sind, bleibt bei beiden Plakaten offen. Dasselbe gilt auch für die Wahlprogramme, in denen der Begriff als mehr oder weniger hohle Phrase benutzt wird, wie das Beispiel des Unterkapitels 2.2.2. „Akzeptanz unserer christlichen, abendländischen Werte $^{\prime 14}$ im Wahlprogramm aus Hessen aus dem Jahre 2018 belegt. Unter dieser Überschrift finden sich lediglich folgende Ausführungen (ungekürzt):

Unabhängig von Herkunft und Glauben ist das Grundgesetz anzuerkennen. Wir sprechen uns gegen Urteile mit Herkunfts- oder Religionsrabatt aus. Die politische Gesinnung darf keinen Einfluss auf die Strafzumessung haben. Wir fordern unter anderem eine Stärkung der Akzeptanz und des Respekts gegenüber unseren Sicherheitsbehörden. (AfD Hessen, 2018, S. 12) 15 $^{15}$

14 Diese Formulierung findet sich ebenso wie die Berufung auf die „christlich-abendländische Kultur" stereotyp in vielen der ausgewerteten Wahlprogramme der AfD (z.B. AfD Niedersachsen, 2017, S. 12, 48).

15 Ähnlich unspezifisch ist der Befund z.B. auch im Text des Wahlprogramms zur Landtagswahl in SchleswigHolstein (AfD Schleswig-Holstein, 2017). 
Fremde werden hier als potenzielle Straftäter und unangepasste, nicht-integrierte Personen angesehen, christliche Werte offenbar mit dem Gehalt des Grundgesetzes gleichgesetzt. Unausgesprochen wird dabei weniger etwas über christliche Werte ausgesagt, als vielmehr eine Antihaltung gegen Fremdes transportiert.

Die Negation überkommener (christlich markierter) Traditionen, aber auch die Bedrohung von Sicherheit wird also auf Fremde (d.h. unausgesprochen: Muslime) fokussiert: So wendet sich die AfD in Niedersachsen gegen die Gefährdung der öffentlichen Ordnung durch „provokatives Verhalten an christlichen Feiertagen und die Okkupation des öffentlichen Raums z. B. durch [...] Gruppen von Migranten" (AfD Niedersachsen, 2017, S. 51).

Bleibt die positive Bezugnahme auf das Christentum und christliche Traditionen und Werte weitgehend unspezifisch und wird lediglich als Gegenposition gegen Fremdes bzw. Muslimisches genutzt, gibt es andererseits durchaus eine negative Rezeption im Hinblick auf Christliches, was sich vor allem in Kritik an der Institution Kirche und am Handeln von Repräsentanten der christlichen Kirchen ausdrückt. So werden im Programm zu Landtagswahl in Niedersachsen 2017 die Kirchen in einer Reihe mit anderen Akteuren des Establishments (tonangebende Klasse der Parteien, Medien, Gewerkschaften) genannt und unterstellt, "Selbstständige, Facharbeiter und Familien“ würden durch sie „vernachlässigt oder geradezu verachtet" (AfD Niedersachsen, 2017, S. 12). Eine wachsende Distanz zwischen den kirchlichen Repräsentanten und der Basis konstatiert auch das bayerische Programm aus dem Jahre 2018 (AfD Bayern, 2018, S. 13). Aufzählungen wie „Altparteien, Kirchen und Gewerkschaften" oder "die Altparteien und die Amtskirchen" zeigen, dass die AfD die Kirchen(leitungen) als Teil der von ihr bekämpften (alten) Eliten sieht (AfD Thüringen, 2019, S. 54). Auf diese Weise werden indirekt auch AfD-kritische Äußerungen der Kirchenleitungen delegitimiert. Zugleich passt diese Sicht in das grundsätzliche Argumentationsmuster rechtspopulistischer Parteien, das eine Kritik an gesellschaftlichen Eliten aus der (tatsächlichen oder vermeintlichen) Entfremdung dieser von der Basis ableitet (Wolf, 2017, S. 12).

Damit besteht der Diskurs zum Christentum in der AfD aus zwei gegenläufigen Teildiskursen: Einerseits gibt es eine Einbettung in einen generellen Identitätsdiskurs mit Christlichem als Teil der Identität des Volkes, andererseits findet eine Abgrenzung von "denen da oben" statt, konkretisiert als Kirchenrepräsentanten als Teil des Establishments. Insofern wird die Positionierung in doppelter Weise in die allgemeinen Narrative des Rechtspopulismus eingebunden. 
Sowohl im Hinblick auf das Christentum als auch und noch deutlich stärker hinsichtlich des Islams operiert die AfD mit kulturellen Stereotypen und Kollektivsymbolen. In beiden Fällen geht es nicht um Glaubensinhalte, sondern vor allem um Äußerliches und vages Gemeinwissen, sei es bei der Berufung auf die unspezifisch bleibenden christlichen Werte, sei es bei der Ablehnung der vermeintlich für den Islam typischen Symbole wie Vollverschleierung und Kopftuch oder die mit inm assoziierte Terrorismus- und Kriminalitätsgefahr.

Offenkundig sind Diskurse zur Religion, speziell zu Islam und Christentum, als Alternative zu liberalen, aber auch objektivierend-wissenschaftlichen Religionsdiskursen angelegt. Dies wird nicht zuletzt daran erkennbar, dass im Rechtspopulismus Vertreter von Religions- und Islamwissenschaft gerne als Feindbild dienen (Mathias, 2018, S. 161). Vielmehr zielt die Begrifflichkeit und Themensetzung auf einen emotionalen bzw. emotionalisierten Zugang ab, der sich vorwiegend an Äußerlichem orientiert.

\section{Regionale Unterschiede}

Wie die AfD in Wahlkämpfen auf das Thema Religion zugreift, ist keineswegs einheitlich. Vielmehr gibt es hier erhebliche Unterschiede. Die Partei modifiziert ihre Position und die Schwerpunkte in Abhängigkeit von den jeweiligen regionalen Spezifika. Insofern reflektieren insbesondere die einzelnen Wahlprogramme zu den Landtagswahlen diese Unterschiede:

So weist das Programm zur Bayerischen Landtagswahl 2019 ein eigenes Unterkapitel mit dem Titel „Trennung von Staat und Religion“ auf (AfD Bayern, 2019, S. 12). Es referiert auf die im Freistaat Bayern vorherrschende konservative Tradition einer engen Bindung von Staat und christlichen Kirchen. Dies findet im politischen Alltag u.a. darin Ausdruck, dass seitens bayerischer Politiker (vor allem der CSU) der Bezug auf christliche Traditionen immer wieder betont wird. Sichtbaren Widerhall dieser Betonung bayerischer Identität als originär christlich geprägt stellte vor wenigen Jahren die Entscheidung der bayerischen Staatsregierung dar, in allen öffentlichen Räumen staatlicher Einrichtungen in Bayern ein Kreuz aufzuhängen ${ }^{16}$. Die Positionierung der AfD gegen diese Liaison von Staat und Kirchen steht im Einklang mit der für den Rechtspopulismus typischen Elitenkritik, (Wolf, 2017, S. 12, 16) die verbunden ist mit einer Ablehnung des überkommenen Systems. Sie mag aber auch Reaktion auf die AfD-kritische Haltung der christlichen Kirchen sein.

$16 \S 28$ Allgemeine Geschäftsordnung für die Behörden des Freistaates Bayern. 
Im Programm zur Wahl zum Abgeordnetenhaus im stark säkularisierten Berlin 2016 hingegen ließ sich kein einziges der gesuchten Lexeme mit christlichem Bezug nachweisen, dafür finden sich immerhin gut zwei Dutzend mit Islambezug (AfD Berlin, 2016). Christliches spielt hier also keine Rolle, eine Auseinandersetzung mit dem Islam in einer stark multikulturell geprägten Stadt allerdings schon.

Ähnlich sind die Befunde für alle ausgewerteten Programme zu Landtagswahlen in den östlichen Bundesländern, die durch die DDR-Vergangenheit starke Entkirchlichungsprozesse durchlaufen haben: Das Programm zur Landtagswahl Mecklenburg-Vorpommern 2016 weist als einziges Lexem mit christlichem Bezug den Begriff Kirchenasyl auf, während zum Islam wenigstens Islam, islamisch, Islamismus und schächten nachweisbar sind (AfD Mecklenburg-Vorpommern, 2016). Insgesamt spielen aber religiöse Aspekte hier ohnehin eine untergeordnete Rolle: Aus der Gesamtmenge von insgesamt 70 verschiedenen religiösen Lexemen, nach denen in den Texten gesucht wurde und die im Gesamtüberblick auch nachweisbar waren, finden sich im besagten Wahlprogramm lediglich fünf und diese zudem auch noch in der Regel als Einzelbelege.

Analoges gilt auch für das Programm aus Sachsen-Anhalt (2016), in dem sich von den gesuchten Lexemen nur neun nachweisen ließen. Lexik und Diskurse mit Religionsbezug spielen hier also ebenfalls eine nur marginale Rolle.

Zumindest aus quantitativer Sicht anders verhält es sich hingegen in den Programmen zu den Landtagswahlen im Freistaat Sachsen (2019), in Brandenburg (2019) und in Thüringen (2019). Im sehr umfangreichen sächsischen Programm stehen einer Referenz auf Christentum und Kirche (Einzelbeleg Kirche) immerhin 27 Belege zum Islam gegenüber (AfD Sachsen, 2019). Dies ist einerseits Ausdruck der säkularisierten Gesellschaft, in der Christliches nur noch randständig ist. Andererseits erfolgt eine Fokussierung auf eine Religion (Islam), die auf Grund der sehr geringen Zahl an Menschen mit Migrationshintergrund im Freistaat Sachsen ${ }^{17}$ jedoch eine noch weitaus geringere

17 Der Ausländeranteil im FS Sachsen betrug nach Angaben des Statistischen Bundesamtes zum 31.12.2019 nur etwa 5.3\% im Vergleich zum bundesdeutschen Durchschnitt von 13.5\% (https://www.destatis.de/DE/ Themen/Gesellschaft-Umwelt/Bevoelkerung/Migration-Integration/Tabellen/auslaendischebevoelkerung-bundeslaender.html). Legt man den erweiterten Begriff von Personen mit Migrationshintergrund zugrunde, so gilt: „Im Jahr 2018 lebten 95,3 Prozent der Personen mit Migrationshintergrund in Westdeutschland und Berlin." (https://www.bpb.de/nachschlagen/zahlen-undfakten/soziale-situation-in-deutschland/61646/migrationshintergrund-i). 
Bedeutung als in den meisten anderen Bundesländern besitzt: Die Zahl der Christen liegt in Sachsen bei etwa 25\%, die der Muslime deutlich unter $1 \%{ }^{18}$.

Nicht ganz so extrem, aber ähnlich deutlich ausgeprägt ist auch das Verhältnis der Belege im Programm aus Thüringen. Hier stehen zwei Belegen von Lexemen mit christlichem Bezug 18 solche mit Referenz auf den Islam gegenüber (AfD Thüringen, 2019). In Brandenburg schließlich beträgt das Verhältnis drei zu zwölf Belege (AfD Brandenburg, 2019). In beiden Bundesländern leben - ähnlich wie in Sachsen - nur sehr wenige Muslime, der Bevölkerungsanteil beträgt schätzungsweise 0,2 \% in Thüringen und $0,1 \%$ in Brandenburg ${ }^{19}$.

Islamisches Leben ist daher in den drei genannten Bundesländern lediglich ein unbedeutendes Randphänomen. Dennoch warnt die AfD vor der Gefahr einer „Herausbildung muslimisch dominierter Parallelgesellschaften“ analog zu den alten Bundesländern (AfD Brandenburg, 2019, S. 58). Es ist also bezeichnend, dass ein gesellschaftliches Randthema wie das Schächten in Sachsen auf einem der Wahlplakate der AfD thematisiert wird. Eine Marginalie wird dabei emotionalisiert und skandalisiert, ohne dass sie für die weit überwiegende Mehrheit der Wähler relevant wäre.

Der Islam-Diskurs ist hier also eine klare Überspitzung und Dramatisierung eines wenig bedeutsamen Themas. Zum Zwecke der Pointierung und Emotionalisierung wird er verknüpft mit einem Sicherheits-, Gesellschafts- und Identitätsdiskurs, indem eine Bedrohung der Gesellschaft und ihrer Identität durch „Parallelgesellschaften“ im Zeichen der Religion identifiziert bzw. konstruiert wird. ${ }^{20}$ Dass Muslimisches fremd ist und bleibt und daher Gegenpol zur von der AfD behaupteten eigenen Identität (d.h. derjenigen der Deutschen) ist, wird nicht nur in der wiederholten Feststellung deutlich, dass der Islam nicht zu Deutschland gehöre ${ }^{21}$, sondern auch in expliziten Sätzen wie: Der "Islam [...] gehört [...] nicht zu unserer Identität" (AfD Sachsen, 2019, S. 38).

18 https:/de.statista.com/statistik/daten/studie/201622/umfrage/religionszugehoerigkeit-der-deutschennach-bundeslaendern/, https://www.slpb.de/themen/gesellschaft/religion/religion-in-sachsen.

19 https://www.muslimisch-in-ostdeutschland.de/regionale-informationen/muslim_innen-inostdeutschland/

20 Eine detaillierte Untersuchung der Sprechakte Drohung und Warnung am Beispiel der AfD-Plakate wäre eine eigene Studie wert. Eine eingehende Analyse, wie dabei Gefahren sprachlich konstruiert werden, kann hier aus Platzgründen nicht geleistet werden.

21 Bisweilen wird dies auch noch für das betreffende Bundesland konkretisiert (z.B. AfD Thüringen, 2019, S. 53; Bremen, 2019, S. 14). 
Insgesamt ist in den Landtagswahlkämpfen trotz aller Unterschiede durchweg eine antiislamische Position als Konstante erkennbar. Unabhängig von einer positiven Identitätsstiftung über eine etwaige Berufung auf christliche Traditionen erfolgt hier vorrangig eine negative Bestimmung über eine Abgrenzung vom Islam, der zum bedrohlich Fremden stilisiert wird.

\section{Fazit}

Die Bezugnahme auf Christentum und Islam erfolgt in den untersuchten AfDProgrammen fast durchweg konfrontativ. Christliches wird mit den Schlagwörtern christlich-abendländische Kultur, christlich-abendländische Werte usw. als Inbegriff des Eigenen, Identitätskonstituierenden gesetzt, und zwar im Kontrast und zur Abwehr des Fremden, das in Form des Islams konkretisiert wird. Es bleibt dabei allerdings unklar, worin sich diese christlich-abendländische Kultur, diese christlich-abendländischen Werte manifestieren. Hinsichtlich der Gegenposition (des Fremden) wird jedoch ein klares Bedrohungsszenario aufgebaut, indem der Islam als eine aggressive Gefahr für angestammte Werte und die eigene (deutsche) Identität dargestellt wird.

Die AfD führt keinen Religions-Diskurs im engeren Sinne: Während die Referenzen auf das Christentum fast durchweg rein summarisch gehalten sind und lediglich instrumentell als Signum für die (vermeintlich oder tatsächlich) bedrohte kulturelle Identität genutzt werden, fokussieren die Bezugnahmen auf den Islam vor allem auf äußere Symbole und eine konservative bis fundamentalistische Form der Religion, um über diese Zuspitzung ein pointiertes und prägnantes Gegenbild (Feindbild) zu erzeugen. Während das Eigene (christlich Geprägte) weitgehend unspezifisch bleibt, wird die andere Position deutlich konkretisiert (islamistischer Terror, Muezzinrufe, Vollverschleierung, Unterdrückung der Frau etc.).

Identitätsstiftung erfolgt hier also indirekt über die Abgrenzung von einem (nur vordergründig von einer Religion definierten) Negativbild. Dies kann als Ausdruck einer Sehnsucht nach einer übersichtlich geordneten eigenen Kulturwelt ohne Multikulturalismus, ohne "fremde" Einflüsse in Form von Migranten, ohne Ängste vor dem Ungewissen und dem Fremden verstanden werden. Auch wenn die eigene Identität weitgehend unbestimmt bleibt, erscheint in der Ablehnung des Anderen eine Angst vor einer gesellschaftlichen Transformation, vor Modernisierung und der damit verbundenen Erschütterung und Veränderung des Vertrauten und Überkommenen. 
Darstellung und Konstruktion von Identität und Alterität sind die zentralen Motive für den Rückgriff der AfD auf Religion. Dies ist ein schon lange allgemein beobachtetes Charakteristikum des Rechtspopulismus:

Rechtspopulistische Politik ist in ihrem Kern Identitätspolitik, eine Politik des unverhandelbaren ,Entweder/Oder', eine Politik des ,Wir oder Die?' [...]. Rechtspopulistische Politik richtet sich primär darauf, bei breiten Bevölkerungsschichten ein ausgeprägtes Differenzbewusstsein gegenüber den ,Anderen' zu kultivieren, ein Bewusstsein, das in dieser Unterscheidung den zentralen gesellschaftlichen und politischen Konflikt verortet. (Geden, 2006, S. 219)

Das dabei erkennbare Ungleichgewicht zwischen Positivem und Negativem zeigt, dass die AfD ihre Identitätsstiftung zumindest in diesem Bereich weniger durch klare Bestimmung des Eigenen als vielmehr durch Negierung und Abgrenzung von Fremdem realisiert.

Eine Auseinandersetzung mit religiösen (Glaubens-)Inhalten findet in keinem Falle statt. Insofern erweist sich der Zugriff auf Religion lediglich als ein Operieren mit einer Hülse, die ein geeignetes Gefäß für die Füllung mit positiven bzw. vor allem negativen Emotionen darstellt.

Begutachtung: Extern begutachtet. Interessenkonflikt: Es besteht kein Interessenkonflikt.

Finanzielle Förderung: Dieser Beitrag entstand im Rahmen des Projektes „Vergleich sprachlicher Strategien des Rechtspopulismus (Deutschland - Österreich - Tschechien - Slowakei): Lexik - Texte - Diskurse" („Komparácia jazykových stratégií pravicového populizmu (Nemecko - Rakúsko - Česko - Slovensko): lexika texty - diskurzy") an der Universität der HI. Cyrill und Method Trnava (APVV-17-0128).

Peer-review: Externally peer-reviewed.

Conflict of Interest: The author has no conflict of interest to declare.

Grant Support: This article was written within the framework of the project "Comparison of linguistic strategies of right-wing populism (Germany - Austria - Czech Republic - Slovakia): lexica - texts - discourses“ („Komparácia jazykových stratégií pravicového populizmu (Nemecko - Rakúsko - Česko - Slovensko): lexika - texty - diskurzy“) at the University of St. Cyril and Methodius Trnava (APVV-17-0128).

\section{Literaturverzeichnis}

AfD Baden-Württemberg (2016). Für unser Land - für unsere Werte. Landtagwahlprogramm 2016. Stuttgart: AfD Landesverband Baden-Württemberg. 
AfD Bayern (2018). Bayern. Aber sicher! Wahlprogramm Landtagswahl Bayern 2018. Nürnberg: AfD Landesverband Bayern.

AfD Berlin (2016). Berlin braucht Blau. Das Wahlprogramm der AfD Berlin. Berlin: AfD Landesverband Berlin.

AfD Brandenburg (2019). Landtagswahlprogramm für Brandenburg 2019. Hol Dir Dein Land zurück! Potsdam: AfD Landesverband Brandenburg.

AfD Bremen (2019). Wahlprogramm zur Bremer Bürgerschaftswahl Mai 2019. Bremen: AfD Landesverband Bremen.

AfD Hessen (2018). Hessen. Aber sicher! Wahlprogramm Landtagswahl Hessen 2018. Frankfurt am Main: AfD Landesverband Hessen.

AfD Mecklenburg-Vorpommern (2016). Wahlprogramm der Alternative für Deutschland MecklenburgVorpommern zur Landtagswahl 2016. Schwerin: AfD Landesverband Mecklenburg-Vorpommern.

AfD Niedersachsen (2017). Landtagswahlprogramm. Der Heimat eine Zukunft geben! [s.I.]: AfD Landesverband Niedersachsen.

AfD Nordrhein-Westfalen (2017). Für unsere Familien und unsere Heimat. Wahlprogramm der AfD für die Bürger Nordrhein-Westfalens. Düsseldorf: AfD Landesverband Nordrhein-Westfalen.

AfD Rheinland-Pfalz (2016). Freiheit, Demokratie und Verantwortung. Mit Herz und Verstand für unser Land. Wahlprogramm des Landesverbandes Rheinland-Pfalz der Alternative für Deutschland zur Landtagswahl 2016. Mainz: AfD Landesverband Rheinland-Pfalz.

AfD Saarland (2017). Landtagswahlprogramm 2017. Saarbrücken: AfD Landesverband Saarland.

AfD Sachsen (2019). Trau Dich Sachsen. Regierungsprogramm der Alternative für Deutschland zur Landtagswahl Sachsen 2019. Dresden: AfD Landesverband Sachsen.

AfD Sachsen-Anhalt (2016). „Die Stimme der Bürger - unser Programm!“ Wahlprogramm. Zur Landtagswahl am 13. März 2016. „Wir für unsere Heimat". Magdeburg: AfD-Landesverband Sachsen-Anhalt.

AfD Schleswig-Holstein (2017). Unser Land, unsere Heimat. Wahlprogramm der AfD Schleswig-Holstein für die Landtagswahl 2017. Kiel: AfD Landesverband Schleswig-Holstein.

AfD Thüringen (2019). Meine Heimat, mein Thüringen. Wahlprogramm der Alternative für Deutschland für die Landtagswahl in Thüringen 2019. Erfurt: AfD Landesverband Thüringen.

Alternative für Deutschland (2017). Programm für Deutschland. Wahlprogramm der Alternative für Deutschland für die Wahl zum Deutschen Bundestag am 24. September 2017. Berlin: AfD.

Alternative für Deutschland (2019). Europawahlprogramm. Programm der Alternative für Deutschland für die Wahl zum 9. Europäischen Parlament 2019. Berlin: AfD.

Butterwegge, C., Hentges, G. \& Wiegel, G. (2018). Rechtspopulisten im Parlament: Polemik, Agitation und Propaganda der AfD. Frankfurt/Main: Westend Verlag.

Demčišák, J. (2019). Zu sprachlichen Strategien und visuellen Symbolen in der politischen Werbung der FPÖ. In E. Kaščáková \& H. Kožaríková (Hrsg.). Forlang. Cudzie jazyky v akademickom prosredí (S. 159-168). Košice: Technická Univerzita v Košiciach. Katedra jazykov. 
Geden, O. (2006). Diskursstrategien im Rechtspopulismus. Freiheitliche Partei Österreichs und Schweizerische Volkspartei zwischen Opposition und Regierungsbeteiligung. Wiesbaden: VS Verlag für Sozialwissenschaften.

Grigat, S. (Hrsg.) (2017). AfD \& FPÖ. Antisemitismus, völkischer Nationalismus und Geschlechterbilder. BadenBaden, Wien: Nomos, Facultas.

Häusler, A. (2017). AfD, Pegida \& Co. Die Formierung einer muslimfeindlichen rechten Bewegung. In P. Antes \& R. Ceylan (Hrsg.). Muslime in Deutschland. Historische Bestandsaufnahme, aktuelle Entwicklungen und zukünftige Forschungsfragen (S. 59-74). Wiesbaden: Springer VS.

Häusler, A. (Hrsg.) (2016). Die Alternative für Deutschland. Programmatik, Entwicklung und politische Verortung. Wiesbaden: Springer VS.

Khodyeyev, I. (2016). Europa in Wahlplakaten. Eine politolinguistische Untersuchung der Wahlplakate als politischer Textsorte bei Europawahlen in der Bundesrepublik Deutschland (1979-2014). Berlin, Mykolajiw: Ilion.

Kranert, M. (2018). Populistische Elemente in den Wahlprogrammen von AfD und UKIP. Aptum. Zeitschrift für Sprachkritik und Sprachkultur 14, 61-77.

Kuhs, J. (Hrsg.) (2018). Bekenntnisse von Christen in der Alternative für Deutschland. Graz: Oxalis-Verlag.

Mathias, A. (2018). „Wir sind das Volk!“. Zu Wortschatz und Argumentation rechtspopulistischer Gruppierungen in Deutschland. Der Sprachdienst 62, 155-167.

Michel, S. \& Pappert, S. (2018a). Wahlplakat-Busting: Kommunikative Spuren der Aneignung von Wahlplakaten im öffentlichen Raum. Fallanalysen - Forschungsfragen - Perspektiven. Ein Werkstattbericht. In Michel, S. Michel \& S. Pappert (Hrsg.). Multimodale Kommunikation in öffentlichen Räumen. Texte und Textsorten zwischen Tradition und Innovation (S. 187-199). Stuttgart: ibidem.

Michel, S. \& Pappert, S. (2018b). Wahlplakat-Busting. Formen und Funktionen einer (neuen) Textmustermischung. Zeitschrift für Angewandte Linguistik 68, 3-33.

Müller, G. (1978). Das Wahlplakat. Pragmatische Untersuchungen zur Sprache in der Politik am Beispiel von Wahlplakaten aus der Weimarer Republik und der Bundesrepublik. Tübingen: Niemeyer.

Müller, G. (2001). Kommunikative und ästhetische Funktionen des Wahlplakats. In J.-F. Leonhard, H.-W Ludwig, D. Schwarze \& E. Straßner (Hrsg.). Medienwissenschaft. Ein Handbuch zur Entwicklung der Medien und Kommunikationsformen. 2. Teilband (S. 1770-1782). Berlin, New York: de Gruyter.

Niehr, T. \& Reissen-Kosch, J. (2018). Volkes Stimme? Zur Sprache des Rechtspopulismus. Berlin: Dudenverlag.

Pappert, S. (2016). Offline-Wahlkampf: Wahlplakate. Aptum. Zeitschrift für Sprachkritik und Sprachkultur 12, 236253.

Pappert, S. (2017). Plakatbusting: Zur Umwandlung von Wahlplakaten in transgressive Sehflächen. In H. Kämper \& M. Wengeler (Hrsg.). Protest - Parteienschelte - Politikverdrossenheit. Politikkritik in der Demokratie (S. 5575). Bremen: Hempen.

Poier, I. M. (2004). Das Wahlplakat als Kommunikationsforum im politischen Prozess. In H. Kopetz, J. Marko \& K. Poier (Hrsg.). Soziokultureller Wandel im Verfassungsstaat. Phänomene politischer Transformation. Festschrift für Wolfgang Mantl zum 65. Geburtstag. Bd. 1 (S. 637-654). Wien, Köln, Graz: Böhlau. 
Püttmann, A. (2017). Was ist die AfD? Und wie als Kirche mit ihr umgehen? In S. Orth \& V. Resing (Hrsg.). AfD, Pegida und Co. Angriff auf die Religion? (S. 36-57). Freiburg, Basel, Wien: Herder.

Schuppener, G. (2018). Phraseologismen auf Wahlplakaten. In A. Gondek, A. Jurasz \& J. Szczęk (Hrsg.). Einblicke und Rückblicke. Beiträge zur deutschen Phraseologie und Parömiologie aus intra- und interlinguistischer Sicht. Bd. 2 (S. 39-57). Baltmannsweiler: Schneider Verlag Hohengehren.

Schuppener, G. (2019). Lexik und Diskurse rechtspopulistischer Wahlwerbung - Plakate der AfD aus den Jahren 2016 bis 2018. Aussiger Beiträge 13, 243-259.

Strube, S. A. (2017). Christliche Unterstützer der AfD. Milieus, Schnittmengen, Allianzen. In S. Orth \& V. Resing (Hrsg.). AfD, Pegida und Co. Angriff auf die Religion? (S. 58-71). Freiburg, Basel, Wien: Herder.

Thielmann, W. (Hrsg.) (2017). Alternative für Christen? Die AfD und ihr gespaltenes Verhältnis zur Religion. Neukirchen-Vluyn: Neukirchener Verlag.

Wolf, T. (2017). Rechtspopulismus. Überblick über Theorie und Praxis. Wiesbaden: Springer VS.

\section{Internet-Quellen}

https://de.statista.com/statistik/daten/studie/201622/umfrage/religionszugehoerigkeit-der-deutschen-nachbundeslaendern/ [28.5.2020]

https://de.wikipedia.org/wiki/Christen_in_der_AfD [28.5.2020]

https://www.bpb.de/nachschlagen/zahlen-und-fakten/soziale-situation-in-deutschland/61646/ migrationshintergrund-i [28.5.2020]

https://www.destatis.de/DE/Themen/Gesellschaft-Umwelt/Bevoelkerung/Migration-Integration/Tabellen/ auslaendische-bevoelkerung-bundeslaender.html [28.5.2020]

https://www.deutschlandfunkkultur.de/die-geschichte-eines-satzes-der-islam-gehoert-zu-deutschland.1895.

de.html?dram:article_id=308696 [8.6.2020]

https://www.ekd.de/ekd-ratsvorsitzender-in-aller-klarheit-gegen-afd-hetze-37096.htm [30.4.2020]

https://www.evangelisch.de/inhalte/156631/11-06-2019/afd-politiker-um-hoecke-werfen-ekd-politisierungvor [30.4.2020]

https://www.muslimisch-in-ostdeutschland.de/regionale-informationen/muslim_innen-in-ostdeutschland/

[2.6.2020]

https://www.nwzonline.de/meinung/berlin-analyse-afd-im-clinch-mit-den-kirchen_a_50,3,1227031897.html

[30.4.2020]

https://www.slpb.de/themen/gesellschaft/religion/religion-in-sachsen [28.5.2020]

https://www.vaticannews.va/de/kirche/news/2019-11/deutschland-katholikenkomitee-widerstand-afd.html

[30.4.2020]

\section{Abbildungsnachweis}

Abb. 1: http://www.afd-pi.de/z_galerie/ [31.1.2019] 
Abb. 2: http://www.afd-pi.de/z_galerie/ [31.1.2019]

Abb. 3: https://www.afdbayern.de/wahlen-2018/themenplakate/ [8.12.2018]

Abb. 4: http://www.gemeinsamfuerdeutschland.de/index.php/landtagswahl-2018 [28.1.2019]

Abb. 5: https://www.designtagebuch.de/von-praegnant-bis-lasch-die-wahlplakate-zur-nrw-landtagswahl-2017 /afd-wahlplakat-nrw-landtagswahl-2017-5/ [28.1.2019]

Abb. 6: http://www.afd-pi.de/z_galerie/ [31.1.2019]

Abb. 7: https://www.eat-drink-think.de/glosse-zum-afd-plakat-der-islam-passt-nicht-zur-deutschen-kueche/ [19.11.2018]

Abb. 8: http://afd.berlin/wahlplakate-2016/ [12.2.2019]

Abb. 9: https://www.facebook.com/AfD.Sachsen/posts/2130076913788148 [20.7.2020] 